\title{
Control of the noncentrosymmetry of thick polymer films by nonresonant all-optical poling
}

$\operatorname{AUTHOR}(\mathrm{S})$ :

Si, Jinhai; Qiu, Jianrong; Hirao, Kazuyuki

\section{CITATION:}

Si, Jinhai ...[et al]. Control of the noncentrosymmetry of thick polymer films by nonresonant all-optical poling. JOURNAL OF APPLIED PHYSICS 2001, 90(10): 4895-4899

\section{ISSUE DATE:}

2001-11-15

URL:

http://hdl.handle.net/2433/39703

\section{RIGHT:}

Copyright 2001 American Institute of Physics. This article may be downloaded for personal use only. Any other use requires prior permission of the author and the American Institute of Physics. 


\title{
Control of the noncentrosymmetry of thick polymer films by nonresonant all-optical poling
}

\author{
Jinhai $\mathrm{Si}^{\mathrm{a})}$ and Jianrong Qiu \\ Photon Craft Project, ICORP, JST, Keihanna Plaza, Super-Laboratory 2-5, 1-7 Hikaridai, Seika-cho, Kyoto \\ 619-0237, Japan \\ Kazuyuki Hirao \\ Division of Material Chemistry, Faculty of Engineering, Kyoto University, Sakyo-ku, Kyoto 606-8501, Japan
}

(Received 18 June 2001; accepted for publication 17 August 2001)

\begin{abstract}
All-optical control of the noncentrosymmetry of azodye-doped polymer films was demonstrated by nonresonant dual-frequency coherent excitation. Three kinds of noncentrosymmetries of the polymer films were optically tailored using appropriate combinations of the writing beam polarizations, linearly polarized, and circularly polarized light. The tensor analysis of the photoinduced noncentrosymmetry was performed by monitoring second harmonic generation (SHG) of the samples. The quadratic dependence of SHG on film thickness was observed, indicating that a $\chi^{(2)}$ grating satisfying the phase-matching condition for SHG was optically induced in the polymer films and that this nonresonant all-optical poling can be used to tailor the symmetry of bulk samples. (C) 2001 American Institute of Physics. [DOI: 10.1063/1.1412571]
\end{abstract}

\section{INTRODUCTION}

Controlling and manipulating the external degrees of freedom of atoms and molecules are a central subject in modern physics and chemistry. In studies of condensed matter, scanning probe microscopes enable selection and manipulation of individual atoms and molecules. ${ }^{1}$ In chemistry researchers have aimed at control of the spatial orientation of molecules because it allows for studies of orientational effects in chemical reactions. ${ }^{2}$ The control can be achieved by applying a strong static electric field for molecules possessing permanent dipole moments, ${ }^{3}$ or by introducing a strong laser field for neutral molecules. ${ }^{4,5}$

Dual-frequency coherent laser excitation at fundamental and second harmonic frequencies has been shown to permit the achievement of large polar orientation efficiency in azodye-doped polymers. ${ }^{6}$ One breakthrough with such an alloptical poling technique is that it leads to an automatic molecular organization with a period satisfying the phasematching conditions for second harmonic generation (SHG). Additionally, using appropriate combinations of the writing beam polarizations, all-optical poling enables full control of the molecular polar order, which permits complete all-optical tailoring of the symmetry of polymer materials. ${ }^{7,8}$ The physical mechanism of all-optical poling consists of two processes: orientational hole burning and reorientation of azodye molecules. ${ }^{9,10}$ Under the phase-coherent excitation of a fundamental light along with its second-harmonic light, orientational hole burning of azodye molecules occurs through the interference of two-photon absorption at the fundamental frequency and one-photon absorption at the doubling frequency. Orientational hole burning is followed by a reverse trans-cis-trans isomerization, which finally leads to

\footnotetext{
a) Author to whom correspondence should be addressed; electronic mail: jhsi@photon.jst.go.jp
}

a net permanent polar orientation of molecules. This technique possesses some of the following advantages: phase matching for SHG can automatically be achieved, and micropatterning of the molecular polar orientation can be simply achieved by scanning the focal area in the polymer sample.

In a resonant optical excitation process, however, it appears to be difficult to control the symmetry of bulk samples using this all-optical poling due to the material absorption, and it is also difficult for the SHG conversion efficiency to benefit from phase matching achieved automatically in large propagation distances. ${ }^{11}$ In 1977, Fiorini et al. reported a nonresonant all-optical poling of bulk rods of poly(polymethylmethacrylate) (PMMA)-grafted para-nitroaniline using a 30 ps pulse laser of a Nd:YAG at 1064 and $532 \mathrm{~nm}^{12}$ Recently, we successfully achieved nonresonant all-optical poling of azodye-doped PMMA films using a femtosecond laser, ${ }^{13}$ in which phase-matched SHG in thick polymer films was first demonstrated at three wavelengths of 1500, 1320, and $1300 \mathrm{~nm}$, respectively. In this article, we experimentally demonstrate optical control of the symmetry of azodyedoped PMMA thick films using the nonresonant opticalpoling technique. During the writing process, samples were irradiated simultaneously by coherent superposition of the $1500 \mathrm{~nm}$ fundamental and $750 \mathrm{~nm}$ second-harmonic light of a femtosecond laser. Three kinds of noncentrosymmetries of the polymer films were optically tailored using appropriate combinations of the writing beam polarizations. The characteristic kinetics of the photoinduced molecular polar orientation and the tensor analysis were performed by monitoring SHG of the films. The quadratic dependence of SHG on film thickness showed that a $\chi^{(2)}$ grating satisfying the phasematching condition for SHG was optically induced in the polymer films, indicating this nonresonant all-optical poling can be used to tailor the symmetry of bulk samples. 

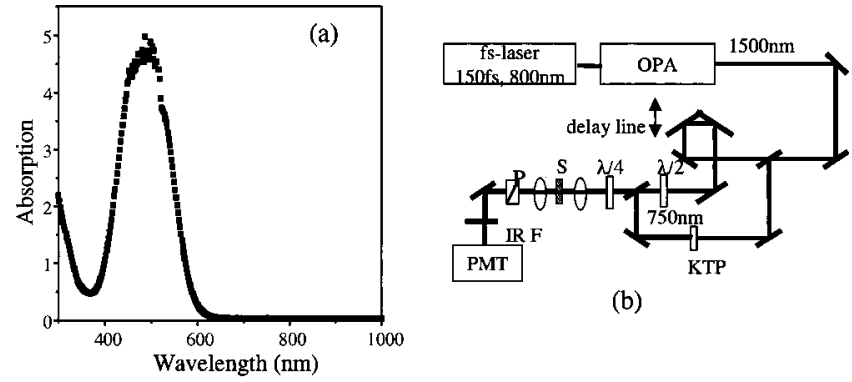

(b)

FIG. 1. Absorption spectrum of an azodye-doped PMMA film (a) and experimental setup (b).

\section{EXPERIMENTS}

The samples in this study were prepared by dissolving to a 15\% mass ratio purified disperse red 1 (DR1) and medium molecular weight PMMA in telrahydorofuran. The films were spin deposited onto glass substrates, and dried for several hours at $160^{\circ} \mathrm{C}$. Films with different thicknesses were prepared by adjusting the viscosity of the solution and the revolutions per minute of the spin coater. According to the absorption spectra of the films shown in Fig. 1(a), we know that the absorption band peaks at $480 \mathrm{~nm}$, and the film sample is almost transparent at wavelengths larger than 650 nm.

As shown in Fig. 1(b), a Ti:sapphire laser system with an optical parametric amplifier (OPA) which emitted 150 fs laser pulses at a repetition rate of $1 \mathrm{kHz}$ was used for alloptical poling of the films, and the wavelength of the light from the OPA could be tuned from 300 to $3000 \mathrm{~nm}$. The average power of the laser was about $50 \mathrm{~mW}$ at wavelengths ranging from 1300 to $1500 \mathrm{~nm}$. A writing beam $\omega$ for alloptical poling was split from the source beam and passed through a time-delay device and a $\lambda / 2$ plate to control the path length and the polarization of the beam, respectively. Another beam separated from the source beam was frequency doubled by a KDP crystal, which served as another writing beam, and is denoted the $2 \omega$ writing beam. The two collinear writing beams whose wavelengths were set at 1500 and $750 \mathrm{~nm}$ were introduced into the film sample through a 6-cm focal-length quartz lens. In order to get circularly polarized writing beams, a dichromatic $\lambda / 4$ plate was placed in front of the lens. We achieved time superposition of pulses between the two writing beams by adjusting the time-delay device and observing the optical Kerr effect of $\mathrm{CS}_{2}$. During the optical-poling process, the two writing beams were introduced simultaneously into the film sample; during the probe, the $2 \omega$ beam was blocked by a shutter and only the $\omega$ beam remained incident. The SHG signal of the $\omega$ beam was detected by a photomultiplier and observed and averaged by an oscilloscope. The $\omega$ beam passing through the sample was blocked by a heat-absorbing filter placed behind the sample, and allows only the SHG signal to pass through it. Typical fluences of the two writing beams at the sample were $\sim 1.5$ $\mathrm{mJ} / \mathrm{cm}^{2}$ for the $\omega$ beam and $0.03 \mathrm{~mJ} / \mathrm{cm}^{2}$ for the $2 \omega$ beam, respectively.

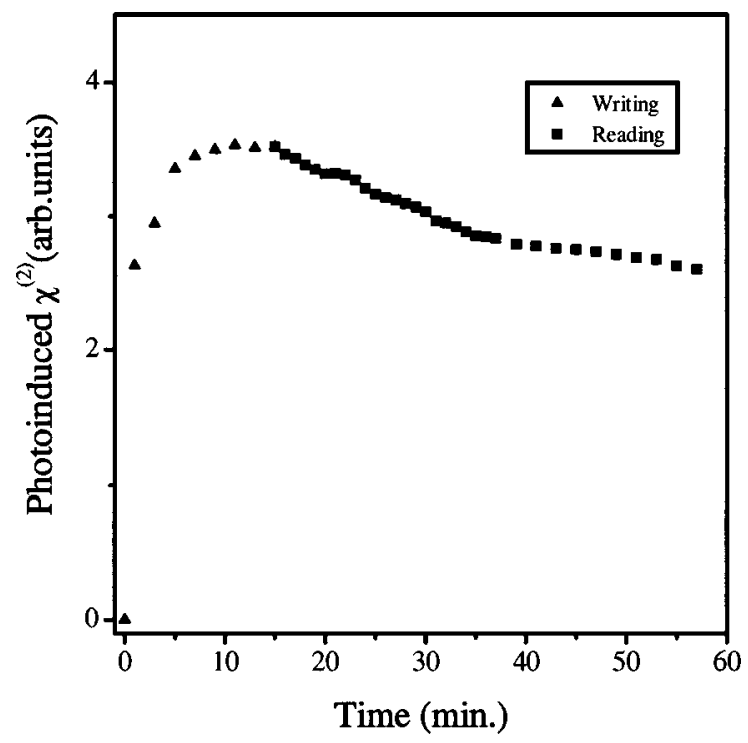

FIG. 2. Growth and decay processes of the photoinduced $\chi^{(2)}$ of a $73 \mu \mathrm{m}$ film.

\section{RESULTS AND DISCUSSION}

\section{A. Dependence of SHG on film thickness}

By adjusting the delay device, we measured the dependence of photoinduced SHG on the temporal correlation of the two writing beams, and observed that all-optical poling of the films could have been performed only when the two writing beams were at a coherent superposition. This indicated that all-optical poling resulted from the coherent excitation of the two writing lights for azodye molecules in the films. Figure 2 shows the growth and decay processes of photoinduced $\chi^{(2)}$ of a film with thickness of $73 \mu \mathrm{m}$. It can be found that the photoinduced $\chi^{(2)}$ reaches its saturation value in $10 \mathrm{~min}$ for the condition described above.

Figure 3 shows the dependence of the SHG intensity

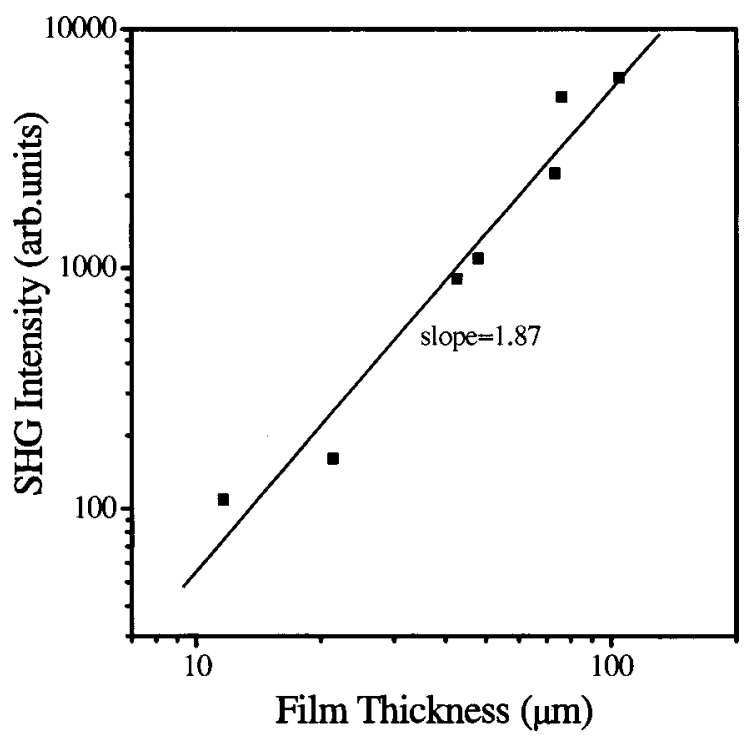

FIG. 3. Dependence of the SHG intensity vs film thickness. Log-log plots with a linear fit of slope near 2 display SHG intensity dependence on the film thickness squared. 
versus film thickness, where both $\omega$ and $2 \omega$ writing beams were $y$ polarized and all of the measurements were made after the photoinduced $\chi^{(2)}$ of the films increased to their saturation values. It can be seen from Fig. 3 that the SHG signals increase proportionally to the square of the film thickness. It is well known that for a thick film with thickness of larger than SHG coherent length (the coherent length of these films is about $12.5 \mu \mathrm{m}$ ), the quadratic thickness dependence of the SHG intensity occurs only in the phasematched condition. A peak conversion efficiency of $2 \%$ was observed for the $105 \mu \mathrm{m}$ film and a fundamental light intensity of $9 \mathrm{GW} / \mathrm{cm}^{2}$ incident on the sample. Obviously, this high conversion efficiency benefitted from quasiphasematched SHG in the thick film. We also observed that the SHG intensity of the films departed from the quadratic length dependence when the thickness of the films was larger than $90 \mu \mathrm{m}$. This probably is due to the group velocity mismatch between the $\omega$ and $2 \omega$ writing beams. The group velocity mismatch causes the $\omega$ writing pulse to displace against the $2 \omega$ writing pulse as they propagate along in the film. This reduces the effective interaction length and decreases the optical-poling efficiency.

\section{B. Control of the noncentrosymmetry of azodye- doped polymers by dual-frequency coherent excitation}

Quantum interference between the writing fields at frequencies $\omega$ and $2 \omega$ results in a selective polar excitation of the individual molecules. ${ }^{7}$ Such a dual-frequency coherent excitation breaks the initial centrosymmety of the material, leaving a new distribution of molecules $N_{p}(M)$ exhibiting polar order.

We let $z$ be the propagation direction and the optical polarization vectors lie in the $x-y$ plane. It is well known that different polarization combinations for the writing beams at frequencies $\omega$ and $2 \omega$ can induce different angular distribution of molecules. We demonstrated control of the angular distribution of azodye molecules in polymers by three different polarization combination as follows: (1) both $\omega$ and $2 \omega$ writing beams were $y$ polarized; (2) the $\omega$ writing beam and the $2 \omega$ writing beam had the same circular polarization; (3) the $\omega$ writing beam and the $2 \omega$ writing beam had opposite circular polarizations.

For cases (1), (2), and (3), the angular distribution of molecules in the plane transverse to the propagation direction can be written, respectively, as ${ }^{14}$

$$
\begin{aligned}
& \frac{d N_{p}}{d \Omega}=\left\{Q+Q^{\prime} \sin ^{2} \Phi\right\}\left\{Q^{\prime \prime} \sin \Phi\right\} \exp (i \Delta k z)+\text { c.c. } \\
& \frac{d N_{p}}{d \Omega}=\left\{Q e^{i \Phi}\right\} \exp (i \Delta k z)+\text { c.c. } \\
& \frac{d N_{p}}{d \Omega}=\left\{Q e^{i 3 \Phi}\right\} \exp (i \Delta k z)+\text { c.c. }
\end{aligned}
$$

where $\Phi$ is the azimuthal angle, and the complex $Q$ s include the radial overlap integral.

Tensor analysis of the photoinduced $\chi^{(2)}$ symmetry of the polymer films was achieved by studying the polar maps
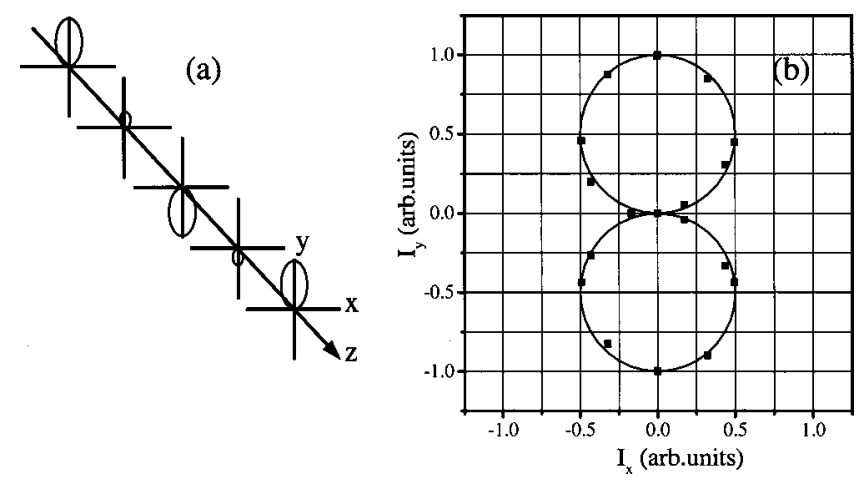

FIG. 4. Photoinduced $\chi^{(2)}$ symmetry for linear writing beam polarizations: (a) schematic of the angular distribution probability of molecules in the $x-y$ plane at different $z$; (b) polar representation of the measured SHG for the $40.5 \mu \mathrm{m}$ film, where the data were obtained by simultaneous rotation of the readout-beam polarization and the analysing polarizer.

of its SHG, and the data were obtained by rotating simultaneously polarization of the linearly polarized readout beam and the analyzing polarizer.

For case (1), Eq. (1a) gives a result as shown in Fig. 4(a), where preferential direction of azodye molecules varies sinusoidally with $z$ between $+y$ and $-y$, and this means that the magnitude of the photoinduced $\chi^{(2)}$ varies sinusoidally with $z$. Figure $4(\mathrm{~b})$ shows a polar map of the measurement for the photoinduced $\chi^{(2)}$ of a $40.5 \mu \mathrm{m}$ thick film. From the figure, we can see that as predicted by Eq. (1a) the photoinduced $\chi^{(2)}$ exhibits axial symmetry along the writing beam polarization direction $(y)$.

The results predicted by Eq. (1b) for case 2 are shown in Fig. 5(a), where preferential direction of molecules rotates in the $x-y$ plane periodically with $z$, and the magnitudes of the photoinduced $\chi^{(2)}$ remain at a constant along $z$. Polar maps of the measured $\chi^{(2)}$ of a $0.1 \mu \mathrm{m}$ thick film and a $40.5 \mu \mathrm{m}$ thick film are shown in Figs. 5(b) and 5(c), respectively. From the figures, we can see that the polar maps of these two films show very different characters: the thin film with a thickness of $0.1 \mu \mathrm{m}$ exhibits a polar map similar to Fig. 4(b). The tensor analysis for the thick film shows that the photoinduced $\chi^{(2)}$ has a radial symmetry because preferential direction of molecules rotates in the $x-y$ plane periodically with $z$. These data are in agreement with the results predicted by Eq. (1b) [see Fig. 5(a)].

Figure 6(a) shows the results predicted by Eq. (1c) for case (3), where perferential direction of molecules with three lobed rotates in the $x-y$ plane periodically with $z$. Polar map of the measured $\chi^{(2)}$ of the $0.1 \mu \mathrm{m}$ thick film is shown in Fig. 6(b). The photoinduced $\chi^{(2)}$ of the $40.5 \mu \mathrm{m}$ thick film has similar radial symmetry as shown in Fig. 5(c).

As described above, in all cases, the photoinduced $\chi^{(2)}$ is sinusoidally modulated along the propagation direction with the period necessary for phase-matched SHG. However, unlike the case of linear writing polarizations resulting in a modulation of the photoinduced $\chi^{(2)}$ amplitude, in the case of circularly polarized writing beams, the only direction of the molecular order $\left(\chi^{(2)}\right.$ axis $)$ is modulated, with no amplitude variation. 

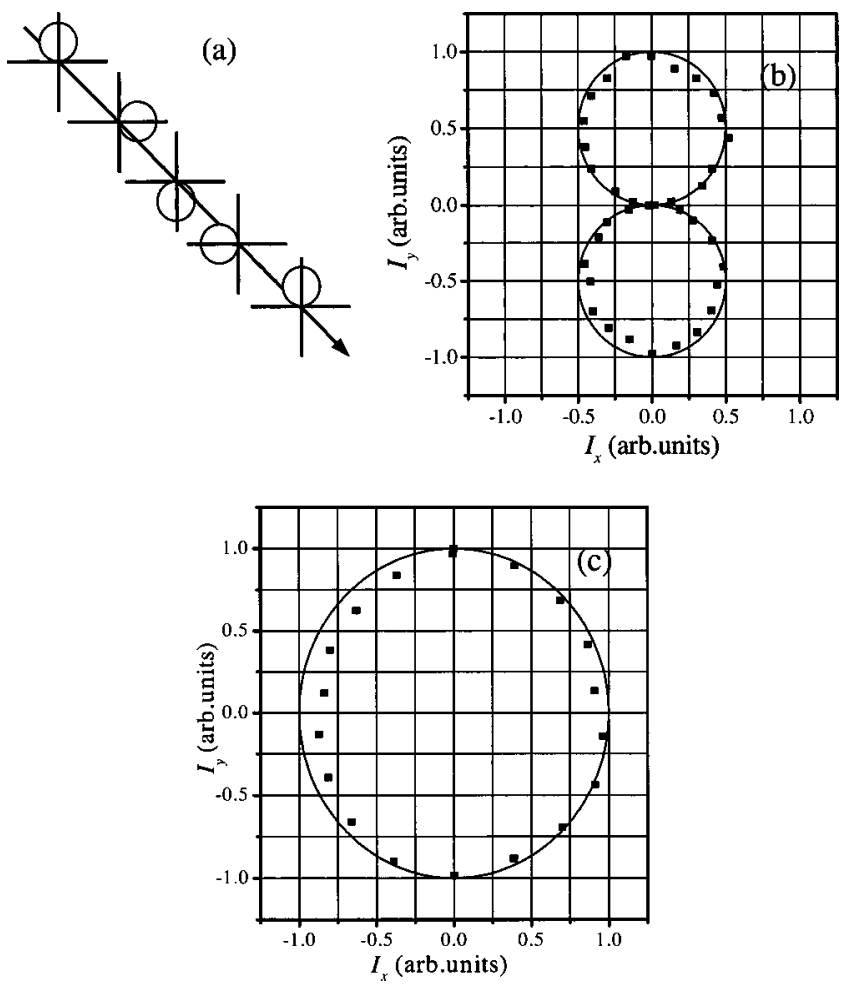

FIG. 5. Photoinduced $\chi^{(2)}$ symmetry for the writing beams with the same circular polarization: (a) schematic of the angular distribution probability of molecules in the $x-y$ plane at different $z$; (b) and (c) polar representations of the measured SHG for the 0.1 and $40.5 \mu \mathrm{m}$ films, respectively.

\section{Enhancement of SHG using circularly polarized writing beams}

We also observed that when polarizations of the writing beams were changed from the linear polarization to the circular polarization the saturated SHG signals were enhanced even at the same readout condition such as the same linear polarization of the readout beam and the same readout power. Case (2) in which both writing beams had the same circular polarization lead to the largest SHG intensity. The saturated SHG intensities for cases (2) and (3) were 2.9 and 1.6 times of that of case (1) in which both $\omega$ and $2 \omega$ writing
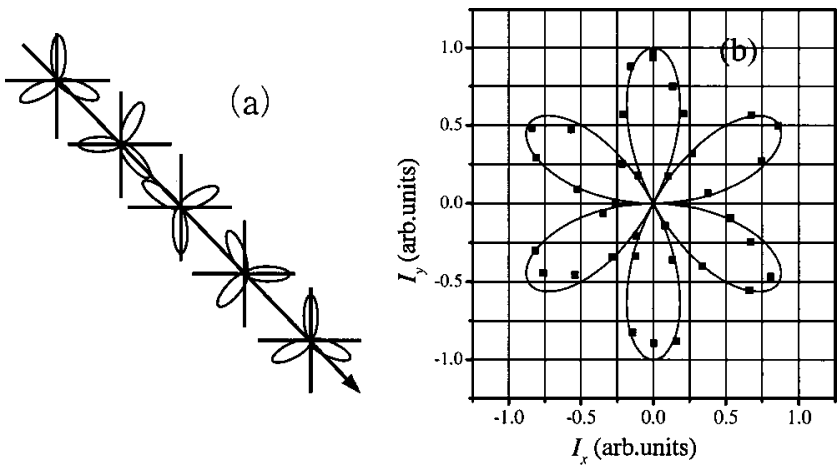

FIG. 6. Photoinduced $\chi^{(2)}$ symmetry for the writing beams with opposite circular polarizations: (a) schematic of the angular distribution probability of molecules in the $x-y$ plane at different $z$; (b) polar representations of the measured SHG for the $0.1 \mu \mathrm{m}$ film. beams were $y$ polarized. In all these measurements, the polarization of the readout beam was linear polarized, and the readout power was kept at $6.5 \mathrm{~mW}$.

According to the tensor analysis described in Sec. III B, the enhancement effect of SHG can be explained as follows: for case (1) optical poling with linear polarized writing beams induced a $\chi^{(2)}$ whose magnitude varied sinusoidally with $z$. In cases (2) and (3), the magnitude of $\chi^{(2)}$ induced by the circular polarized writing beams did not vary with $z$. In other words, compared with case (1) more molecules for cases (2) and (3) can be induced in the polar order and take part in the contribution of $\chi^{(2)}$. In addition, Etile et al. presented a theoretical analysis for all-optical poling. ${ }^{7}$ They concluded that for a circular reading beam polarization the whole $\chi^{(2)}$ is phase matched for SHG, which enables a larger conversion efficiency than in all-optical poling with linear polarization, where only part of $\chi^{(2)}$ is phase matched.

To compare SHG intensities of the films with that of a Y-cut quartz with a thickness of $1 \mathrm{~mm}$, we also measured the SHG intensity ratio for the $73 \mu \mathrm{m}$ film $I_{s} / I_{q}$, where $I_{s}$ and $I_{q}$ are the SHG intensities of the film and the Y-cut quartz for the same readout power, respectively. The maximum of the SHG intensity ratio between the film and the quartz, $I_{s} / I_{q}$, was measured to be 345 for case (1) written by linearly polarized writing beams.

In addition, it should be mentioned that at present we cannot give a clear interpretation for the mechanism of this nonresonant all-optical poling. Here, we only present two possibilities for the mechanism: simultaneous multiphoton excitation and stepwise multiphoton excitation. In simultaneous multiphoton excitation, nonresonant optical poling can be achieved by simultaneous three-photon $(\omega+\omega+\omega)$ and two-photon $(\omega+2 \omega)$ excitation on the same electronic level $S_{1}$. In the stepwise multiphoton excitation, the azodye molecules are first excited from the trans to cis form through multiphoton absorption, such as $\omega+\omega+\omega$ or $\omega+2 \omega$. Then, a resonant all-optical poling can be achieved by simultaneous two-photon $(\omega+\omega)$ and one-photon $(2 \omega)$ excitation on the electronic level $S_{1}^{\prime}$ of the cis form because azodye molecules at the cis form have a redshift absorbtion band compared with the trans form.

\section{CONCLUSION}

In summary, we experimentally demonstrated all-optical control of the noncentrosymmetry of azodye-doped PMMA films by nonresonant dual-frequency coherent excitation. Tailoring of the symmetry of polymer materials was achieved using appropriate combinations of the writing beam polarizations. The tensor analysis of the photoinduced noncentrosymmetry was performed. An enhancement effect of SHG was observed in the films induced by circularly polarized writing beams. The quadratic dependence of SHG on the film thickness showed this nonresonant all-optical poling can be used to tailor the symmetry of bulk samples.

${ }^{1}$ J. A. Stroscio and D. M. Eigler, Science 254, 1319 (1991).

${ }^{2}$ P. Brumer and M. Shapiro, Annu. Rev. Phys. Chem. 43, 257 (1992).

${ }^{3}$ B. Friedrich and D. R. Herschbach, Nature (London) 353, 412 (1991).

${ }^{4}$ H. Sakai, C. P. Safvan, J. J. Larsen, K. M. Hilligsoe, K. Hald, and H. Stapelfeldt, J. Chem. Phys. 110, 10235 (1999). 
${ }^{5}$ D. M. Villeneuve, S. A. Aseyev, P. Dietrich, M. Spanner, M. Yu. Ivanov, and P. B. Corkum, Phys. Rev. Lett. 85, 542 (2000).

${ }^{6}$ C. Fiorini, F. Charra, J-M. Nunzi, and P. Raimond, J. Opt. Soc. Am. B 14, 1984 (1997)

${ }^{7}$ A. Etile, C. Fiorini, F. Charra, and J-M. Nunzi, Phys. Rev. A 56, 3888 (1997).

${ }^{8}$ S. Brasselet, J. Opt. Soc. Am. B 15, 257 (1998).

${ }^{9}$ F. Charra, F. Kajzar, J-M. Nunzi, P. Raimond, and E. Idiart, Opt. Lett. 18, 941 (1993).
${ }^{10}$ F. Charra, F. Devaux, J-M. Nunzi, and P. Raimond, Phys. Rev. Lett. 68, 2440 (1992).

${ }^{11}$ J. Si, K. Kitaoka, T. Mitsuyu, P. Ye, and K. Hirao, J. Appl. Phys. 85, 8018 (1999).

${ }^{12}$ C. Fiorini, F. Charra, P. Raimond, and J-M. Nunzi, Opt. Lett. 22, 1846 (1997).

${ }^{13}$ J. Si, J. Qiu, K. Kitaoka, and K. Hirao, J. Appl. Phys. 89, 2029 (2001).

${ }^{14}$ V. Dominic and J. Feinberg, Phys. Rev. Lett. 71, 3446 (1993). 